\title{
Toward a Culture of Inquiry: Reducing Barriers to Engagement in Assessment
}

\author{
Jeremy Buhler \\ University of British Columbia, Canada
}

\section{Introduction}

Many library assessment professionals are charged with implementing or developing a "culture of assessment" in their organizations. Based on observations at the University of British Columbia (UBC) Library, this case study considers how terms like "assessment" and "culture of assessment" may unintentionally exclude some library employees and limit engagement with assessment activities. Drawing from the literature on inquiry-based learning, the author suggests that, in some contexts, the goals of assessment are served better by fostering a culture of inquiry rather than assessment.

The assessment program at UBC Library strives to create an environment where data is accessible to all library employees and where evidence-based decision-making is the default approach throughout the organization. An assessment team consisting of one librarian, one analyst, and a part-time project assistant works to realize UBC Library's vision for assessment. In this vision,
Staff members at all levels collaborate with the assessment librarian to design focused assessment projects that answer specific questions about library services and user needs. Existing data is accessible, presented with clarity, and used in creative new ways. [...] Library management uses a wide range of metrics and reports, and University administrators are confident that UBC Library decisions are based on the best available evidence. $^{1}$

As this excerpt makes clear, in UBC Library's desired state, assessment is a distributed activity. The assessment team's mandate extends beyond supporting managers and administrators to include developing the data literacy skills and evidence-based practices of a wide range of library employees.

\section{UBC Library assessment: the current state}

Despite this commitment and vision, the overall assessment culture at UBC Library is arguably superficial. There is plenty of support for the assessment program and a widespread belief that assessment is important, but the focus is often the act of assessment (e.g., surveys, focus groups, collecting statistics) rather than on its purpose, to improve library services. While some library units do a good job integrating assessment into their planning and decision-making processes, as a system, UBC Library has room to grow.

To explore how we might improve, I facilitated a focus group with eight UBC library administrators and managers whom I consider some of the library's assessment champions-individuals who support assessment initiatives, understand their practical value, and integrate the findings into their work. The discussion topic was evidence-based practice, and participants were invited to answer two questions: "What are the characteristics of our organization that facilitate evidence based practice?" and "What are the characteristics that inhibit or undermine it?" Participants identified several barriers and enablers that helped me better understand the roles of trust, responsibility, accountability, skills development, and resource availability as they apply to UBC Library.

Important as these themes are, something I had not expected stood out to me during that conversation. Throughout the meeting, some people focused almost exclusively on the "nuts and bolts" of assessment and evidence-based practice, including definitions, methods, and concerns about being sufficiently rigorous. For these participants, the emphasis on how we do assessment work seemed to overshadow the why. This emphasis, along with other questions that arose during the conversation, points to anxiety or uncertainty 
about what counts as measurement, assessment, and evidence-based practice. My experience with assessment consultations at UBC Library also suggests this is a more widespread anxiety in our workplace, not something limited to the focus group participants.

\section{Definitions of measurement and assessment}

Reflecting on this aspect of the conversation, I began to wonder whether the term assessment itself might be a barrier to implementing a healthy "culture of assessment" at UBC Library. The anxiety about what counts as measurement observed in the initial focus group seems due in part to an outsider's misunderstanding. To the degree assessment exists as an area of specialization within the library profession-with its own language, librarians, conferences, and competencies-it also runs the risk of becoming exclusive. If that happens, it should not surprise us when those who consider themselves outsiders turn first to the relative safety of definitions, toolkits, and "best practices" when approaching assessment.

One of the challenges at UBC Library is that by relying on actual or perceived "best practices," some employees have set the bar for assessment so high-in my view, much higher than it needs to be-that to conduct what they consider real assessment would require skills and time they do not have. Similarly, the bar is often set too high for what counts as measurement or evidence itself. Here, again, misunderstanding what constitutes real measurement or real evidence can lead to paralysis, getting in the way of practical day-to-day assessment that we could learn from and act on.

The anxiety about what counts as measurement is something Douglas Hubbard addresses in his book How to Measure Anything: Finding the Value of "Intangibles" in Business. Commonly expressed definitions of measurement imply "that measurement is certainty-an exact quantity with no room for error." Hubbard argues that these definitions are too restrictive, and that if we "incorrectly think that measurement means meeting some nearly unachievable standard of certainty, then few things will seem measurable." ${ }^{2}$ Some of the challenges we face in library assessment come from this misunderstanding about measurement: for someone who maintains such a high bar for measurement, few things will seem able to be assessed.

To counter this misunderstanding, Hubbard draws on practice in the sciences: "For all practical purposes, the scientific crowd treats measurement as a result of observations that quantitatively reduce uncertainty. A mere reduction, not necessarily elimination, of uncertainty will suffice for measurement." ${ }^{3}$ If we accept this definition, a measurement does not need to be perfect to count, it just needs to reduce uncertainty. Does the measurement get you closer than you were to understanding something? Does it reduce the risk of making a costly mistake? If yes, then it counts.

Hubbard's definition is reassuring, possibly even liberating. Why, then, when it has the potential to be liberating, do I sometimes encounter anxiety about embracing Hubbard's definition of measurement when designing assessment projects with UBC colleagues? Or to put the question in more specific terms, why, when I ask colleagues who they need to consult about a project, is the answer so often "everyone"? On one level this should not be surprising: it is in keeping with the perfectionism, attention to detail, and comprehensiveness often identified with the library profession. But to achieve an effective, practical assessment program, it is important to stop striving for completeness in every activity.

\section{A culture of inquiry}

Simply informing people of Hubbard's definition is not enough to change the default trend toward completeness in measurement. Commonly held notions of measurement are too widespread to be dislodged by a simple education campaign. Instead of focusing on the definitions, I started imagining the behaviours I would like to see in our library. What workplace behaviours will contribute most to developing a truly collective culture of assessment at UBC Library?

As I reflected on this question, I realized that I do not care very much whether most of my colleagues are assessing things in their workplace. An assessment that is primarily about the act of assessment-not about making decisions or improving services-has limited value. What I care about deeply is that people 
throughout UBC Library are asking good questions. What I really care about is that they are curious, engaged, and wondering about the environment they work in and the users they serve. If employees are invested in and excited about their questions, the assessment team can harness that curiosity and help them explore the topic and find answers.

The emphasis on asking questions changes the focus from developing a culture of assessment to developing a culture of inquiry. Where the former is specialized and potentially exclusive, the latter taps into experiences of wonder and curiosity that almost anyone can relate to. In the last half of 2018, I hosted a workshop and facilitated several conversations with UBC Library staff about this shift in focus from assessment to inquiry. The idea resonates with many people, but it is too soon to tell what impact-and what unintended consequences-a wholesale effort may have on assessment culture at UBC Library. Even so, there is reason to believe that emphasis on inquiry may motivate people in ways that assessment has not.

When learning about cultures of inquiry, I borrowed heavily from the literature about developing such a culture in the classroom. Like definitions of assessment, definitions of inquiry vary widely and emphasize different aspects of the concept. A relatively straightforward definition from Sharon Friesen's Focus on Inquiry defines it as "a systematic investigation into a problem, issue, topic or idea." ${ }^{4}$ There is considerable overlap with assessment in this definition, but for the purpose of motivating my colleagues and sparking curiosity in the workplace, I favour another definition of inquiry: "a dynamic process of being open to wonder and puzzlement and coming to know and understand the world." 5

It is that second definition, with its emphasis on wonder, puzzlement, and sense-making, that I am calling upon to bring more balance to UBC Library's assessment culture. Terms like assessment and evidence-based practice can be just foreign enough and specialized enough that they become hard for others to relate to unless they are part of the assessment "in crowd." In contrast, terms like wonder and puzzlement might sound unruly, but they suggest an energy that most people can relate to.

\section{Inquiry and motivation}

From another perspective, the conscious shift from assessment to inquiry represents a shift from extrinsic to intrinsic motivation for assessment-related activities. I am not confident that we can develop a sustainable assessment culture at UBC Library if assessment is something we do because we are told to, or just because we know it is good for us. This kind of extrinsic motivation has some value and may be important in the early stages of developing an assessment program, but it can also lead to environments where assessment becomes a formality, lacking the transformative energy and risk that comes from deeper questioning. If the motivation is primarily extrinsic, assessment can easily become a required process that is routine and unrewarding. Emphasizing the role of engaged inquiry, on the other hand, has the potential to tap into intrinsic motivation that is more likely to sustain interest in assessment-related projects.

Drawing once again from the literature about motivation in classroom settings, the concepts of ego involvement and task involvement are also relevant to this discussion. Students who are ego involved "are concerned about demonstrating to others that they have high rather than low ability." As a result, they "tend to opt for tasks that are either too easy (thus ensuring success) or too hard (thus enabling the student to save face)." ${ }^{6}$ For those of us who work in assessment, this behaviour may sound familiar. Replace "students demonstrating high ability" with "libraries demonstrating high return on investment" and you will see why it might be tempting for some libraries to focus their assessment efforts on activities that are likely to have positive outcomes. The way libraries sometimes engage with performance measures and rankings also suggests something akin to ego involvement. One problem with this approach, however, is that we miss out on opportunities to learn.

In contrast to ego involvement is task involvement. Moving back to the classroom model, students who are task-involved tend to prefer realistic challenges, and this is more likely to lead to learning. Success for the task-involved student depends on their own interest, effort, and persistence. ${ }^{7}$ It is hard to imagine library staff engaging in assessment with the same interest and persistence as a task-involved student unless they 
are deeply connected to the question they are exploring. Just knowing you are supposed to measure something is not enough; we need to encourage everyone to ask good questions, and we need to create an environment where it is safe to do so.

\section{Conclusion}

Earlier in this paper, I briefly mentioned the importance of trust in fostering evidence-based practice in our organizations. In the last sections, I have also talked about the energy we might be able to tap into if at least some of our assessment activities are fueled by the intrinsic motivations of wonder and puzzlement. But the wonder in this scenario cannot be artificially planted; it needs to arise from those who are deeply engaged in their work and who are intrinsically motivated to learn more. And we cannot expect energizing, transformative questions to arise unless we collectively create environments where it is safe to ask questions, even if those questions challenge the status quo. In closing, I ask you to consider how you, personally, can help make it safer to question in your own workplace. What power do you have in this regard?

Finally, in addition to being safe to ask questions, it also needs to be rewarding so we do not deplete ourselves in endless questions without the occasional reward of answers. That is where library assessment professionals can step in with their expertise in assessment design: to help identify which questions to pursue at any given time, and to channel the energy of inquiry into methods that are likely to reward the asking.

-Copyright 2019 Jeremy Buhler

\section{Endnotes}

1. Assessment Advisory Group, UBC Library Assessment Plan (Vancouver: University of British Columbia Library, 2014.)

2. Douglas Hubbard, How to Measure Anything: Finding the Value of "Intangibles" in Business, 2nd edition (Hoboken: John Wiley \& Sons, 2010), 22-23.

3. Hubbard, How to Measure Anything. Italics in original.

4. Sharon Friesen, Focus on Inquiry (Calgary: Galileo Educational Network and Werklund School of Education, University of Calgary, 2015), http://inquiry.galileo.org/chl/what-is-inquiry/.

5. "What is Inquiry?" Galileo Educational Network, http://galileo.org/teachers/designinglearning/articles/what-is-inquiry/.

6. Janine Bempechat and Anna Mirny. "Contemporary Theories of Achievement Motivation," in Encyclopedia of Education and Human Development, Stephen J. Farenga and Daniel Ness, eds. (New York: Routledge, 2005), 436.

7. Bempechat and Mirny, "Contemporary Theories," 436. 